\title{
FRONT MOTION IN \\ MULTI-DIMENSIONAL VISCOUS CONSERVATION LAWS WITH STIFF SOURCE TERMS DRIVEN BY MEAN CURVATURE AND VARIATION OF FRONT THICKNESS
}

\author{
BY \\ HAITAO FAN (Department of Mathematics, Georgetown University, Washington, DC 20057) \\ AND
}

SHI JIN (Department of Mathematics, University of Wisconsin, Madison, Wisconsin 53706)

Abstract. The bistable reaction-diffusion-convection equation

$$
\partial_{t} u+\nabla \cdot \mathbf{f}(u)=-\frac{1}{\epsilon} g(u)+\epsilon \Delta u, \quad x \in \mathbb{R}^{n}, u \in \mathbb{R}
$$

is considered. Stationary traveling waves of the above equation are proved to exist when $f(u)$ is symmetric and $g(u)$ is antisymmetric about $u=0$. Solutions of initial value problems tend to almost piecewise constant functions within $O(1) \epsilon$ time. The almost constant pieces are separated by sharp interior layers, called fronts. The motion of these fronts is studied by asymptotic expansion. The equation for the motion of the front is obtained. In the case of $\mathbf{f}=\mathbf{b} u^{2}$ and $g(u)=a u\left(1-u^{2}\right)$, where $\mathbf{b} \in \mathbb{R}^{n}$ and $0<a \in \mathbb{R}$ are constants, the front motion equation takes a more explicit form, showing that the front's speed is

$$
\epsilon\left(\kappa+\frac{\nabla \mu}{\mu} \cdot \mathbf{T}\right),
$$

where $\kappa$ is the mean curvature of the front, $\mu$ is the width of the planar traveling of (1) in the normal direction $\mathbf{n}$ of the front, and $\mathbf{T}$ is a vector tangential to the front. Both $\kappa$ and $\nabla \mu / \mu \cdot \mathbf{T}$ are elliptic operators, contributing to the shrinkage of closed curves. An ellipse in $\mathbb{R}^{2}$ is found to preserve its shape while shrinking.

1. Introduction. Typical reactive flows are governed by Navier-Stokes equations with reaction source terms. These equations have the form

$$
u_{t}+\nabla \cdot \mathbf{f}(u)=\frac{1}{\epsilon} g(u)+\gamma \Delta u, \quad t>0, \mathbf{x} \in \mathbb{R}^{n} .
$$

\footnotetext{
Received October 25, 2001.

2000 Mathematics Subject Classification. Primary 35K57, 35L65, 80A32, 53A10.

Research supported by NSF grant No. DMS 9705732.

E-mail address: fan@math.georgetown.edu

E-mail address: jin@math.wisc.edu
} 
Here $\epsilon>0$ is the reaction time and $\mu$ is the viscosity. We shall consider the scaling $\gamma=\epsilon$ in this paper. We restrict our attention to the bistable type of source term $g(u)$. We assume for convenience that

$$
g( \pm 1)=0, \quad g^{\prime}( \pm 1)<0, \quad g(0)=0, \quad g^{\prime}(0)>0
$$

with no more zeroes. Under the assumption, \pm 1 are the two stable equilibrium points of (1.1). When $\epsilon>0$ is small, we see that the solution of the initial value problem of (1.1) will quickly become almost \pm 1 over subdomains separated by sharp fronts across which $u$ changes from -1 to 1 . We shall study the motion of these fronts in this paper.

The one dimensional version of (1.1) and its inviscid version

$$
u_{t}+f(u)_{x}=\frac{1}{\epsilon} g(u)
$$

are studied by [FH1, FH2, FJT, FJ, Lyb, Mas, Sin1, Sin2]. They considered the large time behavior and $\epsilon \rightarrow 0+$ limits. No study has been done for the multidimensional case of (1.1) yet.

When $\mathbf{f}=0$, the equation (1.1) is a typical reaction-diffusion equation:

$$
u_{t}=\frac{1}{\epsilon} g(u)+\gamma \Delta u, \quad t>0, \mathbf{x} \in \mathbb{R}^{n},
$$

which has been extensively studied. When $\epsilon>0$ is small, the solution of the reactiondiffusion equation tends to a piecewise constant function within $O(1) \epsilon$ time. The constant pieces are separated by layers called fronts. If the two wells of the potential $\int g(u) d u$ are equal, then the front will move at the normal speed $\epsilon \kappa$ where $\kappa$ is the mean curvature of the front. This phenomenon was conjectured by Allen and Cahn $[\mathrm{AC}]$ and justified formally by [Fife, Ca, RSK]. Rigorous results were obtained by [BES, BK, Br, Ch, D, DS, ESS, Il, Son], etc. Among the above papers, the one that is most closely related to this paper is [RSK]. Many of its techniques are used in this paper. For more information about the motion of fronts in reaction-diffusion equations, the reader is referred to the lecture notes [E, Sou] and references cited therein.

The motion by mean curvature when $\mathbf{f}=0$ is due to the interaction of fast reaction and slow diffusion in the reaction-diffusion equation. What is the effect of the convection term $\mathbf{f}$ on the front motion? To answer this question, in this paper, we shall investigate the motion of fronts in (1.1) when $\mathbf{f} \neq 0$. From our earlier results [FJT], we see that in the $\epsilon \rightarrow 0+$ limit, Eq. (1.1) has two types of planar waves: The first kind is the ordinary Lax shock propagating in the direction of the unit vector $\mathbf{n}$, pointing from the 1 to the -1 side, at the speed $\mathbf{n} \cdot(\mathbf{f}(1)-\mathbf{f}(-1)) / 2$ as determined by the Rankin-Hugoniot condition. The second type of wave, called a rarefaction layer, has the speed $-\mathbf{n} \cdot \mathbf{f}^{\prime}(0)$. These wave speeds are the $O(1)$ order approximation of the front propagation speeds in (1.1). They are speeds of planar waves. To get the $O(\epsilon)$ order of the wave speeds and to observe the effect of curvature of the front and variance of thickness of the front in different directions on the front propagation, we set

$$
\mathbf{f}(1)-\mathbf{f}(-1)=\mathbf{f}^{\prime}(0)=0 .
$$

In this paper, we assume that

$$
\mathbf{f}(-u)=\mathbf{f}(u), \quad g(-u)=-g(u) .
$$


Under this assumption, the condition (1.3) is satisfied.

The main results and the organization of this paper is as follows: In Sec. 2, we shall prove that, under the assumption (1.4), solutions of (1.1) with some initial value will converge as $t \rightarrow \infty$ to a planar stationary wave. As a consequence, the existence of stationary planar traveling waves in any direction $\mathbf{n}$ is established. Across these planar waves, $u$ changes from \pm 1 to $\mp 1$. The speed of these planar waves is necessarily 0 . In Sec. 3, we formally derive, via asymptotic expansion, that after $t=O(1) \epsilon$, the domain $\mathbb{R}^{n}$ will be divided into subdomains over which $u \approx \pm 1$. These subdomains are separated by fronts across which $u$ changes from \pm 1 to $\mp 1$. This leads to the investigation of the front propagation in Sec. 4. Suppose we have a front separating the regions $\left\{\mathbf{x} \in \mathbb{R}^{n}: u(\mathbf{x}, t)>0\right\}$ and $\left\{\mathbf{x} \in \mathbb{R}^{n}: u(\mathbf{x}, t)<0\right\}$, over which $u \approx \pm 1$. The location of the front can be denoted as the level curve $\Phi(\mathbf{x}, t, \eta, \epsilon)=0$, where $\eta=\epsilon t$. In Sec. 4 , we shall show, through asymptotic expansions, that $\Phi_{t}=0$, i.e., the front does not move on the $O(1)$ time scale, as expected since the planar waves are stationary. The front will move on the $O\left(\epsilon^{-1}\right)$ time scale. We obtained the partial differential equation relating $\Phi_{\eta}$ and partial derivatives of $\Phi$ up to second order. This equation contains the planar stationary waves $\psi(\mathbf{n} \cdot \mathbf{x} / \epsilon, \mathbf{n})$ of (1.1) in $\mathbf{n}:=\nabla \Phi /|\nabla \Phi|$ direction, determined by

$$
\begin{aligned}
& \mathbf{n} \cdot \mathbf{f}(u) \zeta=g(u)+u_{\zeta \zeta} \\
& u( \pm \infty)= \pm 1 \text { or } \mp 1 .
\end{aligned}
$$

To further understand the behavior of this equation for front motion, we want to study some special cases of $\mathbf{f}$ and $g$ for which explicit solutions of (1.5) can be obtained. For this purpose, we considered in Sec. 5 the case where $\mathbf{f}(u)=\mathbf{b} u^{2}$ and $g(u)=a u\left(1-u^{2}\right)$, where $\mathbf{b}$ is a constant vector in $\mathbb{R}^{n}$ and $a>0$ is a constant. For such $\mathbf{f}$ and $g$, the planar stationary waves $\psi(\mathbf{n} \cdot \mathbf{x} / \epsilon, \mathbf{n})$ of $(1.1)$ in $\mathbf{n}$ direction is

$$
\psi=\tanh (\zeta / \mu)
$$

with

$$
\mu^{-1}=\left(-\mathbf{n} \cdot \mathbf{b} \pm \sqrt{(\mathbf{n} \cdot \mathbf{b})^{2}+2 a}\right) / 2 .
$$

The function $\mu(\mathbf{n})$ is the width of the traveling wave of (1.1) in $\mathbf{n}$ direction. With the help of (1.6) and (1.7), we obtained the explicit expression for the front equation:

$$
\frac{\Phi_{\eta}}{|\nabla \Phi|}=\kappa+\frac{\nabla \mu}{\mu} \cdot \mathbf{T},
$$

where $\kappa$ is the mean curvature of the level curve $\Phi(\mathbf{x}, t, \eta, \epsilon)=$ constant, and the vector $\mathbf{T}:=(\mathbf{b}-(\mathbf{b} \cdot \mathbf{n}) \mathbf{n}) /\left(2 \mu^{-1}+\mathbf{b} \cdot \mathbf{n}\right)$ is in a tangential direction of the level curve. Both $\kappa$ and $\frac{\nabla \mu}{\mu} \cdot \mathbf{T}$ are elliptic operators on $\Phi$. Eq. (1.8) states that the front motion of (1.1) is driven by mean curvature of the front and the relative rate of change in a tangential direction of the thickness of planar traveling waves of (1.1). In the two dimensional case, the contributions of both $\kappa$ and $\frac{\nabla \mu}{\mu} \cdot \mathbf{T}$ are to make a circular front to shrink. An example in $\mathbb{R}^{2}$ is given to show that Eq. (1.8) has an explicit solution representing an elliptic front. This front maintains its shape while shrinking. The time it takes for this elliptic front to shrink to a point and then to disappear is given. We guess that simple closed fronts of other shapes (for example, a circle) will evolve, as $\eta$ increases, towards the shape of this 
ellipse before it shrinks to a point. In Sec. 6, we solve a differential equation to provide its solutions needed in Sec. 4 and Sec. 5 .

2. Planar Traveling Waves of (1.1). In this section, we consider planar traveling waves of (1.1) when $\gamma=A \epsilon$. A planar traveling wave of (1.1) in the direction of the unit vector $\mathbf{n}$ is a solution of (1.1) of the form $u\left(\epsilon^{-1}(\mathbf{x} \cdot \mathbf{n}-c t)\right)$. We see that a planar traveling wave of $(1.1)$ connecting $u_{ \pm}= \pm 1$ or $\mp 1$ must satisfy the following connecting orbit problem of ODE:

$$
\begin{aligned}
& -c u^{\prime}+\mathbf{n} \cdot \mathbf{f}(u)^{\prime}=g(u)+A u^{\prime \prime}, \\
& u( \pm \infty)=u_{ \pm} .
\end{aligned}
$$

For simplicity, we use the following notation

$$
f(u):=\mathbf{n} \cdot \mathbf{f}(u) .
$$

Then the system (2.1) becomes

$$
\begin{aligned}
& -c u^{\prime}+f(u)^{\prime}=g(u)+A u^{\prime \prime}, \\
& u( \pm \infty)=u_{ \pm} .
\end{aligned}
$$

The traveling wave equation of

$$
u_{t}+f(u)_{x}=g(u)+A u_{x x}
$$

is the same as (2.3). To prove the existence of solutions of (2.3), we shall show that the solution of $(2.4)$ with some initial data $u(x, 0)$ will converge to a stationary solution under condition (1.4). Some of the lemmas in this section are proved in our earlier paper $[\mathrm{FJ}]$. However, the proof in [FJ] is for the special case $g(u)=u\left(1-u^{2}\right)$. Here we present a proof that holds for general $g \in C^{1}(\mathbb{R}, \mathbb{R})$. We shall further prove that the speed of traveling waves of (2.4) and hence that of $(2.1)$ is 0 .

LEMma 2.1. Let $u(x, t)$ be the solution of (1.1) with initial data $u(x, 0)$. If $u_{x}(x, 0)<0$ $(>0)$, then $u_{x}(x, t)<0(>0)$.

Proof. Let $v=u_{x}$; then $v$ satisfies

$$
\begin{aligned}
& v_{t}+f^{\prime}(u) v_{x}+f^{\prime \prime}(u) v^{2}=v_{x x}+g^{\prime}(u) v, \\
& v(x, 0)=u_{x}(x, 0)>0(<0) .
\end{aligned}
$$

The maximum principle type of argument applies to $(2.5)$ to yield that $v(x, t)>0(<0)$ if $v(x, 0)>0(<0)$.

In the rest of this section, we assume the initial data satisfies

$$
u_{x}(x, 0)<0 \text {, and }-1 \leq u(x, 0) \leq 1 .
$$

In this case the solution $u(x, t)$ is decreasing. Then the transformation from $(x, t)$ to

$$
\begin{aligned}
& w=u(x, t), \\
& s=t
\end{aligned}
$$


is one-to-one. Then for any smooth function $h(w, s)$, the chain rule reads

$$
\begin{aligned}
& h_{t}=h_{w} u_{t}+h_{s}, \\
& h_{x}=h_{w} u_{x} .
\end{aligned}
$$

Let $v:=u_{x}$. After changing variables according to (2.7) and (2.8), Eqs. (2.5) and (2.6) become

$$
\begin{aligned}
& v_{s}=v^{2}\left[v_{w w}+\left(\frac{g(w)}{v}\right)_{w}-f^{\prime \prime}(w)\right], \quad w \in(-1,1), \\
& v(w, 0)<0 .
\end{aligned}
$$

From Lemma 2.1, we know that the solution of $(2.9)$ satisfies $v(w, s)<0$ for $s>0$.

Lemma 2.2. If $v_{s}(w, 0)>0(<0)$, then $v_{s}(w, s)>0(<0)$ for all $s>0$.

Proof. Taking $\partial / \partial s$ on $(2.9)$, we obtain

$$
\left(v_{s}\right)_{s}=v^{2}\left(v_{s}\right)_{w w}+2 v\left[v_{w w}-f^{\prime \prime}(w)\right] v_{s}-g(w)\left(v_{s}\right)_{w}+g^{\prime}(w)\left(v_{s}\right) .
$$

Again, the maximum principle type of argument applies to (2.10) to yield that if $v_{s}(w, 0)$ $>0(<0)$, then $v_{s}(w, s)>0(<0)$ for all $s>0$.

\section{LEMMA 2.3 .}

(i) Let

$$
u(x, 0)=-\tanh (x / \delta),(\tanh (x / \delta)),
$$

where $\delta>0$ is a constant and $v(w, 0)$ is the function $u_{x}(x, 0)$ with variables $(w, s)$ given in (2.8). Then when $\delta>0$ is small enough, the solution $v$ of (2.9) satisfies $v_{s}(w, s)>0(<0)$ for all $s>0$.

(ii) Let $u(x, 0)$ be the solution of

$$
u_{x}=\frac{1}{\delta_{1}} \frac{g(u)}{u}, \quad u(0,0)=0
$$

If $\delta_{1}>0$ are large enough, then the solution of $(2.9)$ with initial data $v(w, 0)=$ $u_{x}(x(w, 0), 0)$ satisfies $v_{s}(w, s)>0$ for all $s>0$.

(iii) Let $u(x, 0)$ be the solution of

$$
u_{x}=-\frac{1}{\delta_{2}} \frac{g(u)}{u}, \quad u(0,0)=0 .
$$

If $\delta_{2}>0$ are large enough, then the solution of $(2.9)$ with initial data $v(w, 0)=$ $u_{x}(x(w, 0), 0)$ satisfies $v_{s}(w, s)<0$ for all $s>0$.

Proof. (i) We only prove the case where $u(x, 0)=\tanh (x / \delta)$. The other case can be similarly proved by multiplying the minus sign to $v$ since the equation is linear in $v$.

From Lemma 2.2, it suffices to prove that the initial data given by (2.10) satisfies $(2.9)_{2}$ and $v_{s}(w, 0)>0$ for $w \in(-1,1)$. It is easy to see that $v(w, 0)=u_{x}(x, 0)>0$ for $u(x, 0)=\tanh (x / \delta)$. Also, the limiting process $w \rightarrow \pm 1$ corresponds to $x \rightarrow \mp \infty$ and 
hence $v(w \rightarrow \pm 1,0)=0$. We compute straightforwardly to get

$$
\begin{aligned}
v_{s}(w, 0) & =v_{t}+v_{x} \frac{\partial x}{\partial s}=v_{t}-v_{x} \frac{u_{t}}{u_{x}} \\
& =u_{x x x}-u_{x x}^{2} / u_{x}-f^{\prime \prime}(u) u_{x}^{2}+g^{\prime}(u) u_{x}-g(u) u_{x x} / u_{x} \\
& =u_{x}\left(\frac{u_{x x}}{u_{x}}\right)_{x}-f^{\prime \prime}(u) u_{x}^{2}+u_{x}\left(\frac{g(u)}{u_{x}}\right)_{x} \\
& =\operatorname{sech}^{4}\left(\frac{x}{\delta}\right)\left[\frac{2}{\delta^{3}}-\frac{f^{\prime \prime}(u)}{\delta^{2}}\right]+\delta u_{x}\left(\frac{g(u)}{1-u^{2}}\right)_{x} \\
& =\operatorname{sech}^{4}\left(\frac{x}{\delta}\right)\left[\frac{2}{\delta^{3}}-\frac{f^{\prime \prime}(u)}{\delta^{2}}\right]+\delta u_{x}^{2}\left(\frac{g(u)}{1-u^{2}}\right)_{u} \\
& =\operatorname{sech}^{4}\left(\frac{x}{\delta}\right)\left[\frac{2}{\delta^{3}}-\frac{f^{\prime \prime}(u)}{\delta^{2}}+\frac{O(1)}{\delta}\right] .
\end{aligned}
$$

Here we used conditions (1.2) and (1.4) and $u_{x}=\left(1-u^{2}\right) / \delta$. The conclusion follows from Lemma 2.2 immediately.

(ii) Now, the initial data $u(x, 0)$ is determined by

$$
u_{x}=\frac{g(u)}{\delta_{1} u}, \quad u(0,0)=0 .
$$

By the conditions $(1.2)$ on $g(u)$, we see that the range of $u(x, 0)$ is $(-1,1)$ and $u_{x}(x, 0)>0$. The computation, similar to that in the proof of (i), yields that

$$
\begin{aligned}
v_{s}(w, 0) & =u_{x}\left(\frac{u_{x x}}{u_{x}}\right)_{x}-f^{\prime \prime}(u) u_{x}^{2}+u_{x}\left(\frac{g(u)}{u_{x}}\right)_{x} \\
& =\left(\frac{O(1)}{\delta_{1}}+O(1)+\delta_{1}\right) u_{x}^{2} .
\end{aligned}
$$

When $\delta_{1}>0$ is sufficiently large, $v_{s}(w, 0)>0$ and hence $v_{s}(w, s)>0$.

(iii) The proof is almost the same as that of (ii).

Corollary 2.4. Let $v(w, s)$ be the solution of $(2.9)$ with initial data $-\tanh (x / \delta)$ (or $\tanh (x / \delta))$ where $\delta>0$ is sufficiently small. Then $v(w, s) \rightarrow \theta(w)<0(>0)$ for $w \in(-1,1)$ as $s \rightarrow \infty$. Furthermore, the function $\theta(w)$ satisfies

$$
\begin{gathered}
-\infty=\int_{0}^{1} \frac{1}{\theta(w)} d w<\int_{0}^{w} \frac{1}{v(w, 0)} d w<\int_{0}^{w} \frac{1}{v(w, 0)} d w<\int_{0}^{-1} \frac{1}{\theta(w)}=\infty \\
\left(\infty=\int_{0}^{1} \frac{1}{\theta(w)} d w>\int_{0}^{w} \frac{1}{v(w, 0)} d w>\int_{0}^{w} \frac{1}{v(w, 0)} d w>\int_{0}^{-1} \frac{1}{\theta(w)}=-\infty\right) .
\end{gathered}
$$

Proof. We only prove for the case where $v(w, 0)=-\tanh (x / \delta)$. The other case can be proven in the same way. Lemma 2.3 states that the solution of $(2.9)$ with initial data (2.10) with $\delta>0$ sufficiently small satisfies $v_{s}(w, s)>0$ and hence $v(w, s)$ is increasing as $s$ increases. On the other hand, $v$ is also bounded from above by 0 . Therefore, the limit $\lim _{s \rightarrow \infty} v(w, s)=: \theta(w)$ exists for all $w \in(-1,1)$. To prove that $\theta(w)<0$ for $w \in(-1,1)$, we consider the solution $v_{2}$ of $(2.9)$ given in Lemma 2.3(iii) with $\delta_{2}>0$ large enough. This solution satisfies $0>v_{2}(w, 0)>v_{2}(w, s)$ for all $s>0$ and $w \in(-1,1)$. 
We note that the comparison principle holds for Eq. (2.9). To compare the initial datum $v(w, 0)$ and $v_{2}(w, 0)$, we consider the equation defining $v_{2}(w, 0)$ :

$$
\begin{aligned}
& u_{2 x}=-\frac{g\left(u_{2}\right)}{\delta_{2} u_{2}}=-\left(1-u_{2}^{2}\right) \frac{g\left(u_{2}\right)}{\delta_{2} u_{2}\left(1-u_{2}^{2}\right)}, \\
& u_{2}(0)=0 \\
& u_{2}(x(w))=w \\
& v_{2}(w, 0)=u_{2 x}(x(w)) .
\end{aligned}
$$

By condition (1.2), the factor

$$
\left|\frac{g(u)}{\delta_{2} u\left(1-u^{2}\right)}\right| \leq C
$$

for $u \in[-1,1]$. Compare to the equation that the initial data $v(w, 0)$ satisfies:

$$
\begin{aligned}
& u_{x}=-\frac{1-u^{2}}{\delta}, \\
& u(0)=0, \\
& u(x(w))=w, \\
& v(w, 0)=u_{x}(x(w)) .
\end{aligned}
$$

Choosing $\delta_{2}>0$ large enough, we see that when $w=u(x)=u\left(x_{2}\right), 0>v_{2}(w, 0)>$ $v(w, 0)$. Thus, we have

$$
0>v_{2}(w, 0)>v_{2}(w, s)>v(w, s)>v(w, 0)
$$

and hence $0>v_{2}(w, 0) \geq \theta(w)$. Furthermore, the equality

$$
\frac{\partial x}{\partial w}=\frac{1}{u_{x}}
$$

inequalities (2.14), the property of (2.10), and hence that of $v(w, 0)$, implies that

$$
\int_{0}^{1} \frac{1}{\theta(w)} d w<\int_{0}^{w} \frac{1}{v(w, 0)} d w=-\infty
$$

The other half of (2.13) can be proved similarly.

THEOREM 2.5. If $f(u)=f(-u)$, then the solution $u(x, t)$ of $(2.4)$ with initial data

$$
u(x, 0)=-\tanh (x / \delta)(\text { or } \tanh (x / \delta))
$$

with constant $\delta>0$ small enough, converges to a stationary solution of (2.4).

Proof. Again, we present the proof obtained where the initial data is

$$
u(x, 0)=-\tanh (x / \delta) .
$$

The other case can be proved similarly.

From the definition $(2.7)$ of the transformation $(x, t) \mapsto(u, s)$, we see that

$$
\frac{\partial x}{\partial u}=\frac{1}{u_{x}} \text {. }
$$


For the solution given in Corollary 3.2.4, we have

$$
\frac{\partial x}{\partial u}=\frac{1}{\theta(u)}
$$

or equivalently

$$
\lim _{s \rightarrow \infty} x(u, s)=\lim _{s \rightarrow \infty}(x(u, s)-x(0, s))=\int_{0}^{u} \frac{1}{\theta(u)} d u .
$$

The integral in $(2.18)$ is regular for all $w \in(-1,1)$ in view of Corollary 2.4. When $f(u)=f(-u)$, the solution of $(2.4)$ with initial data $(2.16)$ is antisymmetric about the point $x=0$ and hence $x(0, s) \equiv 0$. Then (2.18) yields

$$
x=\lim _{s \rightarrow \infty} x(u, s)=G(u) .
$$

Estimate (2.13) and $\theta(u)<0$ guarantee that for each $x \in \mathbb{R}$, there is a $u(x) \in(-1,1)$, that satisfies (2.19). By definition (2.7), we have

$$
u(x)=u(x(u(x), s), s)=u(x, s)+u_{x}(\eta, s)(x(u(x), s)-x)
$$

for some $\eta$ between $x$ and $x(u(x), s)$. Since $u_{x}$ is bounded as indicated by $(2.14)$, we can take $s \rightarrow \infty$ to obtain

$$
\lim _{s \rightarrow \infty} u(x, s)=u(x)
$$

for all $x \in \mathbb{R}$. It is easy to see that this limit $u(x)$ must be a stationary solution of (2.4).

The above theorem states that there is a stationary traveling wave of $(2.4)$ if $f(u)$ is symmetric and $g$ is antisymmetric about $u=0$. Next, we shall prove that all traveling waves of (2.4) are stationary under this condition.

By straightforward calculation, we obtain the eigenvalues of the linearized equation of (2.4) at $u= \pm 1$. At $u=1$, we have

$$
\lambda_{ \pm}(1)=\frac{1}{2 A}\left[f^{\prime}(1)-c \pm \sqrt{\left(f^{\prime}(1)-c\right)^{2}+8 A}\right]
$$

while at $u=-1$,

$$
\lambda_{ \pm}(-1)=\frac{1}{2 A}\left[f^{\prime}(-1)-c \pm \sqrt{\left(f^{\prime}(-1)-c\right)^{2}+8 A}\right] .
$$

We rewrite $(2.3)$ as

$$
\begin{aligned}
& u^{\prime}=p \\
& A p \frac{d p}{d u}+g(u)=\left(-c+f^{\prime}(u)\right) p \\
& p(u=1)=p(u=-1)=0 .
\end{aligned}
$$

We can compute the value of $d p / d u$ at $u= \pm 1$ to obtain

$$
\left.\frac{d p}{d u}\right|_{u=1}=\lambda_{ \pm}(1)
$$

and

$$
\left.\frac{d p}{d u}\right|_{u=-1}=\lambda_{ \pm}(-1)
$$




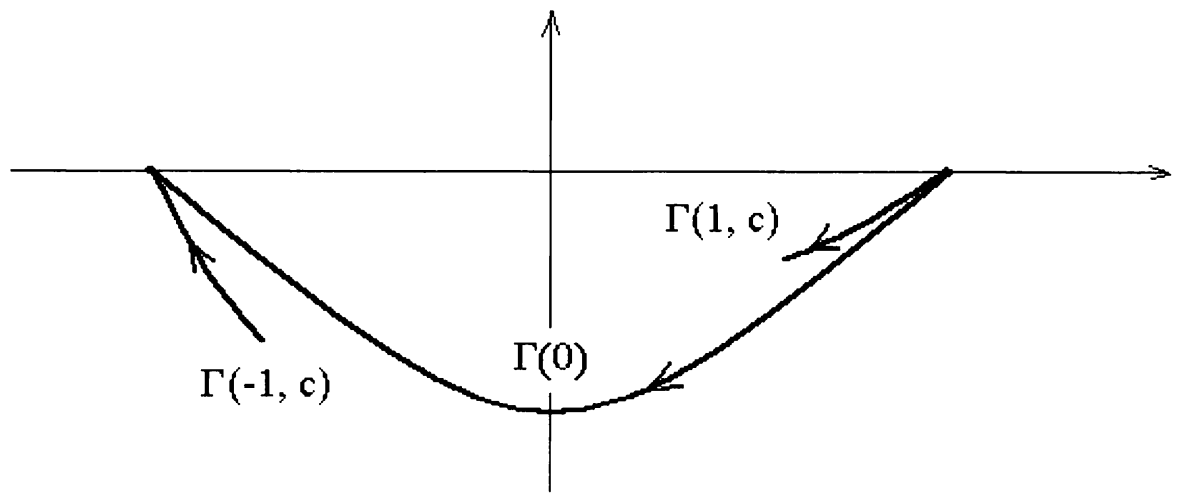

FIG. 2.1

THEOREM 2.6. If $f(u)=f(-u)$ for $u \in \mathbb{R}$, then the speed of traveling waves of (2.4) is necessarily 0 .

Proof. For definiteness, we consider the case where $u_{-}=1$ and $u_{+}=-1$ in (2.3). The other case can be handled similarly.

For a solution of (2.3) to exist, it is necessary and sufficient that an unstable manifold of (2.3) issued from $u_{-}=1$ intersect a stable manifold of (2.3) entering $u_{+}=-1$. The slopes of these manifolds at $u= \pm 1$ are

$$
\begin{gathered}
\left.\frac{d p}{d u}\right|_{u=1}=\lambda_{+}(1)=\frac{1}{2 A}\left[f^{\prime}(1)-c+\sqrt{\left(f^{\prime}(1)-c\right)^{2}+8 A}\right], \\
\left.\frac{d p}{d u}\right|_{u=-1}=\lambda_{-}(-1)=\frac{1}{2 A}\left[f^{\prime}(-1)-c-\sqrt{\left(f^{\prime}(1)-c\right)^{2}+8 A}\right],
\end{gathered}
$$

respectively.

When $c=0$, we know from Theorem 2.5 that an unstable manifold issued from $u_{-}=1$ intersects and hence coincides with a stable manifold entering $u_{+}=-1$. Since the traveling wave given in Theorem 2.5 is monotone, the manifold is in the $p<0$ half plane. We denote this manifold in the $(u, p)$-plane as $\Gamma(0)$.

We see from (2.25) that the slope of the unstable manifold from $\left(u=1, u^{\prime}=0\right)$, denoted as $\Gamma_{+}(1, c)$, decreases as $c$ increases. The slope of the stable manifold, denoted as $\Gamma_{-}(-1, c)$, at $\left(u=-1, u^{\prime}=0\right)$ is also decreasing as $c$ increases. If $c>0$, then the stable manifold at $u=-1$ is below $\Gamma(0)$ near $u=-1$, while the unstable manifold at $u=1$ is above $\Gamma(0)$ near $u=1$. The opposite occurs when $c<0$. Thus, for a connection between $u=-1$ and $u=1$ with $c \neq 0$ to be possible, it is necessary that one of the following two cases hold (see Figure 2.1):

Case 1. $\Gamma_{+}(1, c)$ intersects $\Gamma(0)$.

Case 2. $\Gamma_{+}(1, c)$ crosses $u^{\prime}=0$ from above at $u>1$.

Case 2 is impossible since at $\left(u>1, u^{\prime}=0\right)$,

$$
A u^{\prime \prime}=-g(u)>0
$$

which prevents $\Gamma_{+}(1, c)$ to go below $u^{\prime}=0$. 
Now, to prove this theorem, we only have to prove that Case 1 cannot happen. To this end, we assume the contrary, i.e., $\Gamma(0)$ and $\Gamma_{+}(1, c)$ intersect at some point $\left(u^{*}, p^{*}\right)$. We denote $\Gamma(0)$ by $p_{0}(u)$ and $\Gamma_{+}(1, c)$ by $p_{1}(u)$. Then, we have the following equations from $(2.22)$ :

$$
\begin{aligned}
& A p_{0} \frac{d p_{0}}{d u}+g\left(p_{0}\right)=(-0+f(u)) p_{0}, \\
& A p_{1} \frac{d p_{1}}{d u}+g\left(p_{1}\right)=(-c+f(u)) p_{1} .
\end{aligned}
$$

We further use $\left(u^{*}, p^{*}\right)$ to denote the point of intersection with the maximum $u^{*}$. Then at the point $\left(u^{*}, p^{*}\right)$ and when $c>0$, we have

$$
p_{0}\left(u^{*}\right)=p_{1}\left(u^{*}\right)=p^{*}<0
$$

and

$$
\left.\frac{d p_{0}}{d u}\right|_{u=u^{*}} \leq\left.\frac{d p_{1}}{d u}\right|_{u=u^{*}} .
$$

The difference of $(2.27)$ and (2.28) reads

$$
0 \leq A p^{*}\left[\left.\frac{d p_{0}}{d u}\right|_{u=u^{*}}-\left.\frac{d p_{1}}{d u}\right|_{u=u^{*}}\right]=c p_{1}<0,
$$

which is a contradiction. Similarly, we will also get a contradiction when $c<0$. These contradictions complete the proof.

Corollary 2.7. If $f(u)=f(-u)$ and $g(u)=-g(-u)$, then solutions of $(2.3)$ are antisymmetric about a point $\xi=\xi_{0}$, i.e., $u\left(\xi-\xi_{0}\right)=-u\left(-\left(\xi-\xi_{0}\right)\right)$.

Proof. Assume the contrary, that is, there is a solution of (2.3) that is not antisymmetric, and hence $u^{\prime}$ is not symmetric about any point $\xi$. Then there is a nonsymmetric solution of $(2.22)$, that is

$$
p\left(u_{0}\right) \neq p\left(-u_{0}\right)
$$

for some point $u_{0} \in(-1,1)$. We recall from the last theorem that $c=0$ in $(2.22)$. Then we see that $p(-u)$ is also a solution of (2.22) under the assumptions on $f$ and $g$. If (2.32) held, then $p(u)$ and $p(-u)$ would intersect at some point $u_{1} \in(-1,1)$. However, in our proof of the last theorem, we see that any two manifolds of $(2.22)$ of $(u= \pm 1, p=0)$ either coincide or do not intersect on $(-1,1)$ at all. This contradiction completes our proof.

3. Asymptotic Behavior of $u$. Here we consider the behavior of $u$ away from a front when $\epsilon>0$ is small through asymptotic expansion. To do this, we have to assume functions $f(u)$ and $g(u)$ are smooth enough. We observe that in the region where $u(\mathbf{x}, t, \epsilon)>\delta>0$, the estimate $u(\mathbf{x}, t, \epsilon)=1-O(1) \exp (O(1) t / \epsilon)$ holds. This prompts us to use the "fast variable" $\tau:=t / \epsilon$ and start with the ansatz

$$
u(\mathbf{x}, t, \epsilon)=v_{0}(\mathbf{x}, \tau)+\epsilon v_{1}(\mathbf{x}, \tau)+\epsilon^{2} v_{2}(\mathbf{x}, \tau)+O\left(\epsilon^{3}\right) .
$$


Plugging (3.1) into (1.1) and equating the coefficients of $\epsilon^{-1}, 1$, and $\epsilon$ to zero, we obtain

$$
\begin{gathered}
\partial_{\tau} v_{0}=g\left(v_{0}\right) \\
\partial_{\tau} v_{1}+\nabla \cdot \mathbf{f}\left(v_{0}\right)=g^{\prime}\left(v_{0}\right) v_{1} \\
\partial_{\tau} v_{2}+\nabla \cdot\left(\mathbf{f}^{\prime}\left(v_{0}\right) v_{1}\right)=g^{\prime}\left(v_{0}\right) v_{2}+\frac{1}{2} g^{\prime \prime}\left(v_{0}\right) v_{1}^{2}+\Delta v_{0} .
\end{gathered}
$$

The corresponding initial data for the above equations are

$$
\begin{gathered}
v_{0}(\mathbf{x}, 0)=u(\mathbf{x}, 0), \\
v_{1}(\mathbf{x}, 0)=0, \\
v_{2}(\mathbf{x}, 0)=0
\end{gathered}
$$

respectively. It is clear that (3.2), (3.3), and (3.4) are ordinary differential equations of $v_{0}, v_{1}$, and $v_{2}$ respectively and $\mathbf{x}$ acts only as a parameter.

Now, we study the behavior of $u(\mathbf{x}, t)$ as $\tau \rightarrow \infty$ for $\mathbf{x}$ away from fronts. If we divide $\mathbb{R}^{n}$ into connected components of $\{u(\mathbf{x}, 0)>0\}$ and $\{u(\mathbf{x}, 0) \leq 0\}$, then inside each such component, $v_{0}(\mathbf{x}, \tau) \rightarrow 1$ or -1 , due to the assumption on $g(u),(1.2)$. Similarly, we can prove that $v_{1}$ and $v_{2}$ converge to zero exponentially as $\tau \rightarrow \infty$. From this analysis, we can imagine the picture of $u(\mathbf{x}, t, \epsilon)$ when $\epsilon>0$ is small and $t>0$. At any time $t>0, \mathbb{R}^{n}$ is divided into subdomains inside which $u(\mathbf{x}, t, \epsilon)$ is close to 1 or -1 . Near the boundaries of these subdomains, there are sharp layers, called fronts, across which $u$ changes from 1 to -1 . Then, the characterization of the behavior of $u(\mathbf{x}, t, \epsilon)$ is reduced to that of these sharp layers.

In the next section, we shall investigate the behavior of these fronts.

4. Derivation of the Equation for the Front Motion. In this section, we shall formally derive the motion equation for fronts of (1.1) through asymptotic expansions.

It is clear that the ansatz (3.1) is no longer useful near the fronts since it requires some smoothness uniform in $\epsilon>0$ to be valid. Thus, we shall introduce a new ansatz near a front.

We consider the front $\Gamma_{0}$ in $\mathbb{R}^{n}$ that separates the subdomains $\{u(\mathbf{x}, 0)>0\}$ and $\{u(\mathbf{x}, 0)<0\}$. The equation of this front is $\phi_{0}(\mathbf{x})=0$. The front at time $t$ evolved from $\Gamma_{0}$ is denoted as $\Gamma(t)$. Suppose that the front $\Gamma(t)$ can be represented by a function $\phi(\mathbf{x}, t, \eta)$ with

$$
\eta=\epsilon t
$$

and

$$
\phi(\mathbf{x}, 0, \eta)=\phi_{0}(\mathbf{x}) .
$$

The layer around $\Gamma(t)$ is expected to be of width $\epsilon$. Thus we introduce the stretched spatial variable

$$
y:=\epsilon^{-1} \phi(\mathbf{x}, t, \eta)
$$

We introduce the following ansatz for $u(\mathbf{x}, t, \epsilon)$ around the front $\Gamma(t)$ :

$$
u(\mathbf{x}, t, \epsilon)=u_{0}(y, \mathbf{x}, \tau, t, \eta, \epsilon)+\epsilon u_{1}(y, \mathbf{x}, \tau, t, \eta, \epsilon)+O\left(\epsilon^{2}\right) .
$$


Here $\tau=t / \epsilon$ is defined in Sec. 3. Putting (4.4) into (1.1) and equating the coefficients of $\epsilon^{-1}$ and $\epsilon^{0}$, we obtain

$$
\begin{aligned}
\partial_{\tau} u_{0}+\phi_{t} \partial_{y} u_{0}+\left(\nabla \phi \cdot \mathbf{f}^{\prime}\left(u_{0}\right)\right) \partial_{y} u_{0}=g\left(u_{0}\right)+(\nabla \phi)^{2} \partial_{y}^{2} u_{0}, \\
\partial_{\tau} u_{1}+\left(\phi_{t}+\nabla \phi \cdot \mathbf{f}^{\prime}\left(u_{0}\right)\right) \partial_{y} u_{1} \\
+\left(\nabla \phi \cdot \mathbf{f}^{\prime \prime}\left(u_{0}\right) \partial_{y} u_{0}-g^{\prime}\left(u_{0}\right)\right) u_{1}-(\nabla \phi)^{2} \partial_{y}^{2} u_{1} \\
\quad=-\partial_{t} u_{0}-\nabla \cdot \mathbf{f}\left(u_{0}\right)+2 \nabla\left(\partial_{y} u_{0}\right) \cdot \nabla \phi+\nabla^{2} \phi \partial_{y} u_{0}-\phi_{\eta} \partial_{y} u_{0}
\end{aligned}
$$

Equation (4.5) is a parabolic partial differential equation with variables $\tau>0$ and $y \in \mathbb{R}^{n}$ with parameters $\mathbf{x}, t, \eta$. To uniquely determine $u_{0}$, we need the initial data $u_{0}(y, \mathbf{x}, \tau=0, t, \eta, \epsilon)$. Consider the coordinate system $\mathbf{x}=(\xi, z)$ where $\xi$ is the signed distance from the point $\mathbf{x}$ to the $n-1$ dimensional surface $\Gamma_{0}$ and $z$ is the $n-1$ dimensional coordinate system on $\Gamma_{0}$. According to the definition of $y,(4.3)$, we have

$$
\epsilon y=\phi_{0}(\mathbf{x})=\phi_{0}(\xi, z)=\phi(0, z)+\xi \partial_{\xi} \phi_{0}(0, z)+O\left(\xi^{2}\right)=\xi \partial_{\xi} \phi_{0}(0, z)+O\left(\xi^{2}\right),
$$

where, in the last step, we used that along $\Gamma_{0}, \phi_{0}(\mathbf{x})=\phi_{0}(0, z)=0$. Let the initial data of $(1.1)$ be $u(\mathbf{x}, 0, \epsilon)=a(\mathbf{x})$. Then from (4.4) and (4.7) we have the initial data for $u_{0}$ :

$$
u_{0}(y, 0, z, \tau=0, t, \eta, \epsilon)=a(0, z)+\epsilon y \frac{\partial_{\xi} a(0, z)}{\partial_{\xi} \phi_{0}(0, z)} .
$$

Similar statements hold for $u_{1}$.

When $\tau \rightarrow \infty$, it is expected that the solution $u_{0}$ of (4.5) approaches to a traveling wave. So, we assume that

$$
u_{0}(y, \mathbf{x}, \tau, t, \eta, \epsilon) \sim \psi(y-c \tau, \mathbf{x}, t, \eta),
$$

where $\psi$ is a traveling wave of (4.5) with speed $c$. The function $\psi$ satisfies the traveling wave equation of $(4.5)$ :

$$
\begin{aligned}
& \left(\phi_{t}-c+\nabla \phi \cdot f^{\prime}(\psi)\right) \psi^{\prime}=g(\psi)+(\nabla \phi)^{2} \psi^{\prime \prime}, \\
& \psi( \pm \infty)=u_{ \pm}
\end{aligned}
$$

where $u_{ \pm}$are the two stable equilibria of (1.1).

In Sec. 2, we proved that the traveling wave equation

$$
\begin{aligned}
& \left(-s+F^{\prime}(\psi)\right) \psi^{\prime}=g(\psi)+A \psi^{\prime \prime}, \\
& \psi( \pm \infty)=u_{ \pm}
\end{aligned}
$$

has a solution when $F(u)$ is symmetric and $g(u)$ is anti-symmetric about $u=0$. The speed $s$ must be zero and the solution is anti-symmetric around a point $\xi=\xi_{0}$. Applying this result to (4.10), we see that

$$
c-\phi_{t}=0
$$

and hence

$$
\begin{aligned}
& \left(\nabla \phi \cdot f^{\prime}(\psi)\right) \psi^{\prime}=g(\psi)+(\nabla \phi)^{2} \psi^{\prime \prime}, \\
& \psi( \pm \infty)=u_{ \pm} .
\end{aligned}
$$

From the definition of $y,(4.3)$, we can see that a fixed point in the $\mathbf{x}$-coordinate system travels at a speed $\phi_{t} / \epsilon$ relative to the $y$-coordinate system. The traveling wave of (4.5) travels at a speed $c / \epsilon$ relative to the $y$-coordinate system. Then the speed of this 
traveling wave relative to the $\mathbf{x}$-coordinate system is $\left(c-\phi_{t}\right) / \epsilon$ which is 0 according to (4.12). In other words, the front $\Gamma(t)$ is the level curve

$$
\Phi:=\phi(\mathbf{x}, t, \epsilon)-c t=0,
$$

which does not move on the $t$ time scale. Furthermore, (4.12) shows that $\nabla \phi$ is independent of $t$ and therefore the traveling wave equation (4.10) and hence (4.13) are independent of $t: \psi=\psi(\xi, \mathbf{x})$.

Now, we shall find the motion of the front on the $\eta$ time scale. To this end, we consider Eq. (4.6) for the next order of approximation $u_{1}$. We assume that as $\tau \rightarrow \infty$, $u_{1}$ approaches a traveling wave of (4.6):

$$
u_{1} \rightarrow \omega(y-c \tau, \mathbf{x}, t, \eta)
$$

The traveling wave equation of (4.6) is

$$
\begin{gathered}
\left(-c+\phi_{t}+\nabla \phi \cdot \mathbf{f}^{\prime}(\psi)-g^{\prime}(\psi)\right) \omega^{\prime}+\left(\nabla \phi \cdot \mathbf{f}^{\prime \prime}(\psi)\right) \psi^{\prime} \omega-(\nabla \phi)^{2} \omega^{\prime \prime} \\
=-\nabla \cdot \mathbf{f}(\psi)+2 \nabla \psi^{\prime} \cdot \nabla \phi+\psi^{\prime} \nabla^{2} \phi-\psi^{\prime} \phi_{\eta} .
\end{gathered}
$$

Now, we use $\phi_{t}-c=0$ and (4.10) to obtain

$$
\begin{aligned}
& {\left[\left(\nabla \phi \cdot \mathbf{f}^{\prime}\right) \omega\right]^{\prime}-g^{\prime}(\psi) \omega-(\nabla \phi)^{2} \omega^{\prime \prime}} \\
& \quad=-\nabla \cdot \mathbf{f}(\psi)+2 \nabla \psi^{\prime} \cdot \nabla \phi+\psi^{\prime} \nabla^{2} \phi-\psi^{\prime} \phi_{\eta} .
\end{aligned}
$$

We consider the adjoint equation for the left hand side of (4.16):

$$
(\nabla \phi)^{2} \psi_{1}^{\prime \prime}+\left(\nabla \phi \cdot \mathbf{f}^{\prime}(\psi)\right) \psi_{1}^{\prime}+g^{\prime}(\psi) \psi_{1}=0 .
$$

It is closely related to the equation for $\psi$, (4.13). Taking derivative on (4.13), we get

$$
(\nabla \phi)^{2}\left(\psi_{2}\right)^{\prime \prime}-\left(\left(\nabla \phi \cdot \mathbf{f}^{\prime}(\psi)\right) \psi_{2}\right)^{\prime}+g^{\prime}(\psi) \psi_{2}=0
$$

with $\psi_{2}=\psi^{\prime}$. It is the adjoint equation of (4.17). Since $\psi$ is monotone, the solution $\psi_{2}=\psi^{\prime}$ of $(4.18)$ satisfies $\psi_{2} \neq 0$. We shall see from Theorem 6.1 in Sec. 6 later that the general solution of $(4.17)$ is

$\psi_{1}=\psi^{\prime}\left[C_{2} \exp \left(\int_{0}^{\xi} \frac{-\nabla \phi \cdot f^{\prime}(\psi)}{|\nabla \phi|^{2}} d \theta\right)+\int_{0}^{\xi} \frac{C_{1}}{|\nabla \phi|^{2} \psi^{\prime 2}} \exp \left(\int_{\theta_{1}}^{\xi} \frac{-\nabla \phi \cdot f^{\prime}(\psi)}{|\nabla \phi|^{2}} d \theta_{2}\right) d \theta_{1}\right]$,

where $C_{1}$ and $C_{2}$ are any constants. We choose

$$
\psi_{1}=\psi^{\prime} \exp \left(\int_{0}^{\xi} \frac{-\nabla \phi \cdot f^{\prime}(\psi)}{|\nabla \phi|^{2}} d \theta\right)=: \psi^{\prime}(\xi) W(\xi) .
$$

A straightforward calculation on the decay rate of $\psi$, the solution of (4.13), and that of (4.19) at $\xi= \pm \infty$ shows that $\psi^{\prime} \psi_{1}$ decays at $\xi= \pm \infty$ exponentially. We multiply (4.16) by $\psi_{1}$ given in (4.19) and integrate by parts on the left hand side to obtain

$$
\begin{aligned}
\phi_{\eta} & =\nabla^{2} \phi+\frac{\int_{-\infty}^{\infty}\left[-\nabla \cdot \mathbf{f}(\psi)+2 \nabla \phi \cdot \nabla \psi^{\prime}\right] \psi_{1} d \xi}{\int_{-\infty}^{\infty} \psi^{\prime} \psi_{1} d \xi} \\
& =\nabla^{2} \phi+\frac{\int_{-\infty}^{\infty}\left[-\nabla \cdot \mathbf{f}(\psi) \psi^{\prime} W-\nabla \phi \cdot \psi^{\prime 2} \nabla W\right] d \xi}{\int_{-\infty}^{\infty} \psi^{\prime 2} W d \xi}+\nabla \phi \cdot \frac{\nabla \int_{-\infty}^{\infty} \psi^{\prime 2} W d \xi}{\int_{-\infty}^{\infty} \psi^{\prime 2} W d \xi} .
\end{aligned}
$$


To further clarify the dependence of $\psi$ and $\psi_{1}$ on $\mathbf{x}$, we perform the transformation

$$
\zeta:=\frac{\xi}{|\nabla \phi|}
$$

in $(4.13)$ to yicld

$$
\mathbf{n} \cdot \mathbf{f}(\psi)_{\zeta}=g(\psi)+\psi_{\zeta \zeta}
$$

where

$$
\mathbf{n}:=\frac{\nabla \phi}{|\nabla \phi|}
$$

is the unit normal vector of level curves of $\phi$. We do the same on (4.17) to get

$$
\mathbf{n} \cdot \mathbf{f}^{\prime}(\psi) \psi_{1 \zeta}+g^{\prime}(\psi) \psi_{1}+\psi_{1 \zeta \zeta}=0 \text {. }
$$

From (4.22-4.24), we can see that $\psi$ and $\psi_{1}$ only depend on $\zeta$ and $\mathbf{n}(\mathbf{x})$. In fact, the function $\psi(\zeta, \mathbf{n}(\mathbf{x}))$ is the traveling wave of (1.1) in the direction $\mathbf{n}$. In this new variable, $\psi_{1}$ in (4.19) has the form

where

$$
\psi_{1}=\frac{1}{|\nabla \phi|} \psi_{\zeta}(\zeta, \mathbf{n}) W(\zeta, \mathbf{n})
$$

$$
W=\exp \left(-\mathbf{n} \cdot \int_{0}^{\zeta} \mathbf{f}^{\prime}(\psi(\theta, \mathbf{n})) d \theta\right) .
$$

We note that the gradient $\nabla$ in (4.18) is $\nabla_{\mathbf{x}}$ with $\xi$ fixed, denoted more precisely by $\left.\nabla\right|_{\xi}$. If we want to use the new variable $(\zeta, \mathbf{x})=(\xi /|\nabla \phi|, \mathbf{x})$ in $(4.18)$, we have to use the chain rule

$$
\begin{aligned}
\left.\nabla\right|_{\xi} v & =\left.\nabla\right|_{\zeta} v+\left.\nabla\right|_{\xi}(\zeta) \partial_{\zeta} v \\
& =\left.\nabla\right|_{\zeta} v+\zeta|\nabla \phi| \nabla_{\mathbf{x}}\left(\frac{1}{|\nabla \phi|}\right) \partial_{\zeta} v
\end{aligned}
$$

in (4.20). This leads us to

$$
\begin{aligned}
\phi_{\eta}=\nabla^{2} \phi & -\nabla \phi \cdot \frac{\nabla|\nabla \phi|}{|\nabla \phi|}+\nabla \phi \cdot \frac{\nabla \int_{-\infty}^{\infty} \psi_{\zeta}^{2} W d \zeta}{\int_{-\infty}^{\infty} \psi_{\zeta}^{2} W d \zeta} \\
& -\frac{\nabla \phi}{\int_{-\infty}^{\infty} \psi_{\zeta}^{2} W d \zeta} \cdot \int_{-\infty}^{\infty} \psi_{\zeta}^{2}\left[\nabla W+|\nabla \phi| \nabla\left(\frac{1}{|\nabla \phi|}\right) W_{\zeta} \zeta\right] d \zeta \\
& -\frac{|\nabla \phi|}{\int_{-\infty}^{\infty} \psi_{\zeta}^{2} W d \zeta} \int_{-\infty}^{\infty} \psi_{\zeta} W\left[\nabla \cdot \mathbf{f}+|\nabla \phi| \nabla\left(\frac{1}{|\nabla \phi|}\right) \cdot \mathbf{f}(\psi)_{\zeta} \zeta\right] d \zeta .
\end{aligned}
$$

We note that the first two terms on the right hand side of (4.27) are

$$
|\nabla \phi| \nabla \cdot \frac{\nabla \phi}{|\nabla \phi|}=|\nabla \phi| \kappa_{\phi}
$$

where $\kappa_{\phi}$ is the mean curvature of the level curve of $\phi$. Therefore, the equation of motion for $\phi,(4.27)$, takes the form

$$
\begin{array}{r}
\phi_{\eta}=|\nabla \phi| \kappa+\nabla \phi \cdot \frac{\int_{-\infty}^{\infty}\left(\nabla \psi_{\zeta}^{2}\right) W d \zeta}{\int_{-\infty}^{\infty} \psi_{\zeta}^{2} W d \zeta}+\mathbf{n} \cdot \nabla(|\nabla \phi|) \frac{\int_{-\infty}^{\infty} \psi_{\zeta}^{2} W_{\zeta} \zeta d \zeta}{\int_{-\infty}^{\infty} \psi_{\zeta}^{2} W d \zeta} \\
-\frac{|\nabla \phi|}{\int_{-\infty}^{\infty} \psi_{\zeta}^{2} W d \zeta} \int_{-\infty}^{\infty} \psi_{\zeta} W\left[\nabla \cdot \mathbf{f}+|\nabla \phi| \nabla\left(\frac{1}{|\nabla \phi|}\right) \cdot \mathbf{f}(\psi)_{\zeta} \zeta\right] d \zeta .
\end{array}
$$


When $\mathbf{f}=0$, the function $\psi$ determined by (4.22) is independent of $\mathbf{x}$, and $W=1$. Then Eq. (4.28) reduces to the well-known motion by mean curvature equation

$$
\phi_{\eta}=|\nabla \phi| \kappa_{\phi}
$$

when $\mathbf{f}=0$.

To further simplify (4.28), we let the function $\phi(\mathbf{x}, t, \eta)=d(\mathbf{x}, t, \eta)$, the distance of the point $\mathbf{x}$ to the front. Then the front is denoted by the level curve $d(\mathbf{x}, t, \eta)=0$. With this choice of $\phi$, we have

$$
\mathbf{n}=\nabla \phi,|\nabla \phi| \equiv 1
$$

Thus, Eq. (4.28) is simplified to

$$
d_{\eta}=\kappa+\frac{\int_{-\infty}^{\infty}\left[\mathbf{n} \cdot\left(\nabla \psi_{\zeta}^{2}\right)-\psi_{\zeta} \nabla \cdot \mathbf{f}(\psi)\right] W d \zeta}{\int_{-\infty}^{\infty} \psi_{\zeta}^{2} W d \zeta}
$$

5. Some Special Cases Where (4.22) and (4.24) Have Exact Solutions. To investigate the behavior of the front propagation equation (4.28) further, it is better that we have exact solutions of (4.22) and (4.24) for some special $\mathbf{f}(u)$ and $g(u)$. Here, we assume

$$
g(u)=a u\left(1-u^{2}\right)
$$

and

$$
\mathbf{f}(u)=\mathbf{b} u^{2}
$$

where $a$ is a constant and $\mathbf{b} \in \mathbb{R}^{n}$ is a constant vector. For definiteness, we shall assume

$$
a \geq 0 \text {. }
$$

Then, Eqs. (4.22) take the form

$$
\begin{aligned}
& 2 b \psi \psi^{\prime}=a \psi\left(1-\psi^{2}\right)+\psi^{\prime \prime} \\
& \psi( \pm \infty)= \pm 1(\text { or } \mp 1)
\end{aligned}
$$

where

$$
b:=\mathbf{n} \cdot \mathbf{b} .
$$

It is easy to check that

$$
\psi(\zeta)=\tanh (\delta \zeta)
$$

with

$$
\delta:=\frac{1}{2}\left(-b \pm \sqrt{b^{2}+2 a}\right)
$$

are solutions of (5.4).

Under the choice of (5.1) and (5.2) for $\mathbf{f}$ and $g$, Eq. (4.24) becomes

$$
\psi_{1}^{\prime \prime}+2 b \psi \psi_{1}+g^{\prime}(\psi) \psi_{1}=0 \text {. }
$$

The solution of (5.6) chosen for deriving (4.28) is

$$
\psi_{1}=\psi^{\prime} W
$$

with

$$
W(\zeta)=\exp \left(-2 b \int_{0}^{\zeta} \psi(\theta) d \zeta\right)
$$


From (5.5), we find that

$$
W(\zeta)=\operatorname{sech}^{2 b / \delta}(\delta \zeta)
$$

Now, we plug the expressions (5.9) and (5.5) into (4.28) to see what (4.28) becomes under assumptions (5.1) and (5.2). We write (4.28) as

$$
\phi_{\eta}=|\nabla \phi| \kappa+I I+I I I+I V .
$$

We compute the term $I I$

$$
I I:=\nabla \phi \cdot \frac{\int_{-\infty}^{\infty}\left(\nabla \psi_{\zeta}^{2}\right) W d \zeta}{\int_{-\infty}^{\infty} \psi_{\zeta}^{2} W d \zeta}
$$

as follows:

$$
\begin{gathered}
\int_{-\infty}^{\infty} \psi_{\zeta}^{2} W d \zeta=\delta^{2} \int_{-\infty}^{\infty} \operatorname{sech}^{4+2 b / \delta}(\delta \zeta) d \zeta \\
=\delta \int_{-\infty}^{\infty} \operatorname{sech}^{4+2 b / \delta}(\theta) d \theta
\end{gathered}
$$

where $\theta=\delta \zeta$,

$$
\begin{aligned}
\int_{-\infty}^{\infty} & \left(\nabla \psi_{\zeta}^{2}\right) W d \zeta \\
& =2 \nabla \delta \int_{-\infty}^{\infty}\left(\operatorname{sech}^{4+2 b / \delta} \theta-2 \theta \operatorname{sech}^{4+2 b / \delta} \theta \tanh \theta\right) d \theta \\
& =2 \nabla \delta \int_{-\infty}^{\infty} \operatorname{sech}^{4+2 b / \delta} \theta d \theta+4 \nabla \delta \int_{-\infty}^{\infty} \theta \operatorname{sech}^{3+2 b / \delta} \theta d(\operatorname{sech} \theta) \\
& =\frac{2 \delta+2 b}{2 \delta+b} \nabla \delta \int_{-\infty}^{\infty} \operatorname{sech}^{4+2 b / \delta} \theta d \theta
\end{aligned}
$$

Hence we have

$$
I I=\frac{2 \delta+2 b}{2 \delta+b} \nabla \phi \cdot \frac{\nabla \delta}{\delta}
$$

Similarly, we can obtain

$$
I I I:=\frac{\nabla \phi}{|\nabla \phi|} \cdot \nabla(|\nabla \phi|) \frac{\int_{-\infty}^{\infty} \psi_{\zeta}^{2} W_{\zeta} \zeta d \zeta}{\int_{-\infty}^{\infty} \psi_{\zeta}^{2} W d \zeta}=-\frac{b}{2 \delta+b} \frac{\nabla \phi}{|\nabla \phi|} \cdot \nabla(|\nabla \phi|)
$$

and

$$
\begin{aligned}
I V & :=-\frac{|\nabla \phi|}{\int_{-\infty}^{\infty} \psi_{\zeta}^{2} W d \zeta} \int_{-\infty}^{\infty} \psi_{\zeta} W\left[\nabla \cdot \mathbf{f}+|\nabla \phi| \nabla\left(\frac{1}{|\nabla \phi|}\right) \cdot \mathbf{f}(\psi)_{\zeta} \zeta\right] d \zeta \\
& =-\frac{|\nabla \phi|}{\int_{-\infty}^{\infty} \psi_{\zeta}^{2} W d \zeta} \int_{-\infty}^{\infty} \zeta \psi_{\zeta}^{2} W f^{\prime}(\psi) \cdot\left(\frac{\nabla \delta}{\delta}-\frac{\nabla|\nabla \phi|}{|\nabla \phi|}\right) d \zeta \\
& =-\frac{|\nabla \phi|}{2 \delta+b}\left(\frac{\nabla \delta}{\delta}-\frac{\nabla|\nabla \phi|}{|\nabla \phi|}\right) \cdot \mathbf{b} .
\end{aligned}
$$

Plugging (5.14-5.16) into (5.10), we obtain

$$
\frac{\phi_{\eta}}{|\nabla \phi|}=\kappa+\frac{\nabla \delta}{\delta} \cdot\left(\frac{(2 \delta+2 b) \mathbf{n}-\mathbf{b}}{2 \delta+b}\right)-\frac{\nabla|\nabla \phi|}{|\nabla \phi|} \cdot\left(\frac{b \mathbf{n}-\mathbf{b}}{2 \delta+b}\right) .
$$


With the notation

$$
\mathbf{T}:=\frac{\mathbf{b}-b \mathbf{n}}{2 \delta+b}
$$

Eq. (5.17) becomes

$$
\frac{\phi_{\eta}}{|\nabla \phi|}=\kappa+\frac{\nabla \delta}{\delta} \cdot(\mathbf{n}-\mathbf{T})+\frac{\nabla|\nabla \phi|}{|\nabla \phi|} \cdot \mathbf{T} .
$$

Recalling that $b=\mathbf{n} \cdot \mathbf{b}$ and $\|\mathbf{n}\|=1$, we see that $\mathbf{n} \cdot \mathbf{T}=0$. We recall that $\mathbf{n}=\nabla \phi /|\nabla \phi|$ is the unit normal of the level curve $\phi=C$. Thus, $\mathbf{T}$ is in the tangent direction of the level curve. We claim that

$$
\frac{\nabla \delta}{\delta} \cdot \mathbf{n}+\frac{\nabla|\nabla \phi|}{|\nabla \phi|} \cdot \mathbf{T}=0 .
$$

Indeed, we further compute $\nabla \delta$ defined in (5.5b) to get

$$
\frac{\nabla \delta}{\delta}=\frac{-1}{2 \delta+b} \nabla b
$$

In the remainder of this section, we use the convention that summation is taken for repeated indices. Using $(4.23),(5.4 \mathrm{~b})$, and (5.18) in $(5.20)$, we obtain

$$
\begin{aligned}
& \frac{-1}{2 \delta+b} \nabla b \cdot \mathbf{n}-\frac{\nabla|\nabla \phi|}{|\nabla \phi|} \cdot \frac{\mathbf{b}-b \mathbf{n}}{2 \delta+b} \\
& =\frac{-|\nabla \phi|^{-1}}{2 \delta+b}\left[n_{k} b_{j}\left(\phi_{x_{j} x_{k}}-n_{j} n_{l} \phi_{x_{k} x_{l}}\right)+n_{j} \phi_{x_{j} x_{k}}\left(b_{l} n_{l} n_{k}-b_{k}\right)\right] \\
& =\frac{-|\nabla \phi|^{-1}}{2 \delta+b}\left[n_{k} b_{j} \phi_{x_{j} x_{k}}-b_{k} n_{j} \phi_{x_{j} x_{k}}-n_{k} b_{j} n_{j} n_{l} \phi_{x_{k} x_{l}}+n_{j} b_{l} n_{l} n_{k} \phi_{x_{j} x_{k}}\right] \\
& =0
\end{aligned}
$$

as desired. Thus, we can further simplify Eq. (5.19) as

$$
\frac{\phi_{\eta}}{|\nabla \phi|}=\kappa+\frac{\nabla \delta^{-1}}{\delta^{-1}} \cdot \mathbf{T}
$$

Recalling (5.5), we see that $\delta^{-1}$ is the width of the traveling wave of (1.1) in the $\mathbf{n}$ direction. Then the last term in Eq. (5.22) represents the relative rate of change of the thickness of the front in the tangential direction $\mathbf{T}$ of the level curve $\phi=$ constant.

Since the original equation (1.1) is of parabolic type, we expect Eq. (5.19) for its front is also of parabolic type. We prove this in the following theorem:

Theorem 5.1.

(i) The operator

$$
\kappa=\nabla \cdot \frac{\nabla \phi}{|\nabla \phi|}
$$

in (5.22) is elliptic.

(ii) The operator

$$
\frac{\nabla \delta^{-1}}{\delta^{-1}} \cdot \mathbf{T}
$$

in (5.22) is elliptic. 
Proof. (i) Although $\kappa$ is well known to be an elliptic operator, we still present the proof since we will use it later. We compute $\kappa$ to get

$$
\begin{aligned}
\kappa & =\frac{|\nabla \phi| \nabla^{2} \phi-\nabla \phi \cdot \nabla|\nabla \phi|}{|\nabla \phi|^{2}} \\
& =\frac{|\nabla \phi| \nabla^{2} \phi-\phi_{x_{j}} \phi_{x_{k}} \phi_{x_{k} x_{j}} /|\nabla \phi|}{|\nabla \phi|^{2}} .
\end{aligned}
$$

We replace $\phi_{x_{j} x k}$ in the above by $\lambda_{j} \lambda_{k}$, use $\mathbf{n}=\nabla \phi /|\nabla \phi|$, and apply Schwarz inequality; then the operator $\kappa$ becomes

$$
\frac{\lambda_{j} \lambda_{j}-n_{j} n_{k} \lambda_{j} \lambda_{k}}{|\nabla \phi|}=|\nabla \phi|^{-1}\left((\Lambda, \Lambda)-(\Lambda, \mathbf{n})^{2}\right) \geq 0
$$

where $\Lambda$ is the vector $\left(\lambda_{1}, \lambda_{2}, \ldots, \lambda_{n}\right)$. The equality in (5.23) holds if and only if $\Lambda=$ $\|\Lambda\| \mathbf{n}$. This shows that $\kappa$ is an elliptic operator.

(ii) We further compute $\nabla \delta$ defined in $(5.5 \mathrm{~b})$ to get

$$
\begin{aligned}
\frac{\nabla \delta}{\delta} \cdot \mathbf{T} & =\frac{-1}{2 \delta+b} \nabla b \cdot \mathbf{T} \\
& =\frac{-1}{2 \delta+b} T_{k} b_{j} \partial_{x_{k}} n_{j} \\
& =\frac{-1}{2 \delta+b} T_{k} b_{j}\left(\phi_{x_{j} x_{k}}-n_{j} n_{l} \phi_{x_{l} x_{k}}\right)|\nabla \phi|^{-1} .
\end{aligned}
$$

Replacing $\phi_{x_{j} x_{k}}$ by $\lambda_{j} \lambda_{k}$ and using (5.18) in (5.24), we see that (5.24) becomes

$$
\begin{gathered}
\left(\frac{-1}{2 \delta+b}\right)^{2}|\nabla \phi|^{-1} b_{j}\left(b_{k}-b_{i} n_{i} n_{k}\right)\left(\lambda_{j} \lambda_{k}-n_{j} n_{l} \lambda_{l} \lambda_{k}\right) \\
=\left(\frac{-1}{2 \delta+b}\right)^{2}|\nabla \phi|^{-1}\left(b_{k} \lambda_{k}-b_{i} n_{i} n_{k} \lambda_{k}\right)\left(\lambda_{j} b_{j}-n_{j} b_{j} n_{l} \lambda_{l}\right) \\
=\left(\frac{-1}{2 \delta+b}\right)^{2}|\nabla \phi|^{-1}((\mathbf{b} \cdot \Lambda)-(\mathbf{b} \cdot \mathbf{n})(\mathbf{n} \cdot \Lambda))^{2} \geq 0
\end{gathered}
$$

with equality holds if and only if parts of $\mathbf{b}$ and $\Lambda$ perpendicular to $\mathbf{n}$ are perpendicular to each other: $(\mathbf{b}-(\mathbf{b} \cdot \mathbf{n}) \mathbf{n}) \cdot(\Lambda-(\Lambda \cdot \mathbf{n}) \mathbf{n})=0$. The estimate $(5.25)$ completes the proof. 0 .

REMARK. Eq. (5.22) is degenerate since when $\mathbf{n}=\Lambda /\|\Lambda\|$, both (5.25) and (5.23) are

REMARK. From the above theorem, we see that the convection term of $(1.1), \nabla \cdot \mathbf{f}(u)$, also contributes to the parabolicity of the front motion equation.

Now, we consider the evolution of a front that is a circle in $\mathbb{R}^{2}$ at initial time under Eq. (5.22). Take $\phi(x, y, 0)=x^{2} / 2+y^{2} / 2-r^{2}$ as the initial data where $r>0$ is a constant. We can calculate to get that

and

$$
\kappa=\frac{1}{|\nabla \phi|}>0
$$

$$
-\frac{\nabla \delta}{\delta} \cdot \mathbf{T}=\frac{1}{(2 \delta+b)^{2}}|\nabla \phi|^{-1}\left(\mathbf{b} \cdot \mathbf{b}-(\mathbf{b} \cdot \mathbf{n})^{2}\right) \geq 0
$$


We see from $(5.22)$ that $\phi_{\eta}(x, y, 0)>0$. We then expect that the front represented by $\phi=0$ shrinks as $\eta$ increases. We also see that the circular front shrinks fastest when the normal direction of the front $\mathbf{n}$ is perpendicular to the vector $\mathbf{b}$ in the function $\mathbf{f},(5.2)$, and slowest when $\mathbf{n}$ is parallel to $\mathbf{b}$. This suggests that some elliptic front may preserve its shape as it shrinks. The following example shows that it is indeed the case.

Example. Assume $\mathbf{b}=\left(b_{1}, 0\right)$. Let the initial data for $(5.22)$ be

$$
\phi(x, y, 0)=\frac{x^{2}}{a_{1}^{2}}+\frac{y^{2}}{a_{2}^{2}}-r_{0}^{2} .
$$

If

then the solution of $(5.22)$ is

$$
a_{1}=a_{2} \sqrt{\frac{2 a+b_{1}^{2}}{2 a}},
$$

$$
\phi(x, y, \eta)=\frac{x^{2}}{a_{1}^{2}}+\frac{y^{2}}{a_{2}^{2}}-(r(\eta))^{2},
$$

where

$$
r(\eta)=\sqrt{-a_{1}^{2} a_{2}^{-2} \eta+r_{0}^{2}}
$$

Thus, the front which is at $\phi=0$ will maintain the shape of the ellipse of (5.30) while shrinking as indicated by (5.31). At the rescaled time $\eta=r_{0}^{2} a_{1}^{-2} a_{2}^{2}$, the ellipse will shrink to a point and then disappear.

Proof. By straightforward calculation, using the ansatz (5.30), we see that

$$
\begin{aligned}
& \kappa=\frac{1}{a_{1}^{2} a_{2}^{2}|\nabla \phi|^{3}}\left(\frac{x^{2}}{a_{1}^{2}}+\frac{y^{2}}{a_{2}^{2}}\right) \\
&-\frac{\nabla \delta}{\delta} \cdot \mathbf{T}=\frac{1}{(2 \delta+b)^{2}|\nabla \phi|}\left(b_{k}-(\mathbf{b} \cdot \mathbf{n}) n_{k}\right) b_{j}\left(\phi_{x_{j} x_{k}}-n_{j} n_{l} \phi x_{l} x_{k}\right) \\
&=\frac{1}{(2 \delta+b)^{2}|\nabla \phi|}\left(b_{k}-(\mathbf{b} \cdot \mathbf{n}) n_{k}\right) b_{j}\left(\delta_{j k} a_{j}^{-2}-n_{j} n_{l} \delta_{l k} a_{k}^{-2}\right) \\
&=\frac{1}{(2 \delta+b)^{2}|\nabla \phi|} a_{k}^{-2}\left(b_{k}-(\mathbf{b} \cdot \mathbf{n}) n_{k}\right)^{2} \\
&=\frac{b_{1}^{2} y^{2}}{(2 \delta+b)^{2} a_{1}^{2} a_{2}^{6}|\nabla \phi|^{5}}\left(\frac{x^{2}}{a_{1}^{2}}+\frac{y^{2}}{a_{2}^{2}}\right) .
\end{aligned}
$$

To determine $r(\eta)$, we plug (5.33), (5.32) into (5.22) and use (5.5b) to obtain

$$
\begin{aligned}
\phi_{\eta} & =\frac{1}{a_{1}^{2} a_{2}^{2}|\nabla \phi|^{2}}\left[1+\frac{b_{1}^{2} y^{2}}{|\nabla \phi|^{2}\left(b^{2} n_{1}+2 a\right) a_{2}^{4}}\right]\left(\frac{x^{2}}{a_{1}^{2}}+\frac{y^{2}}{a_{2}^{2}}\right) \\
& =\frac{b^{2}+2 a}{a_{1}^{2} a_{2}^{2}} \frac{1}{\frac{\left(2 a+b^{2}\right) x^{2}}{a_{1}^{4}}+\frac{2 a y^{2}}{a_{2}^{4}}}\left(\frac{x^{2}}{a_{1}^{2}}+\frac{y^{2}}{a_{2}^{2}}\right) .
\end{aligned}
$$

When (5.29) holds, the above equality yields that

$$
\phi_{\eta}=-\left(r^{2}\right)_{\eta}=a_{1}^{2} a_{2}^{-2}
$$

and hence

as desired.

$$
r(\eta)=\sqrt{-a_{1}^{2} a_{2}^{-2} \eta+r_{0}^{2}}
$$


6. Solutions of (4.24). In this section, we shall find solutions of (4.24). This can be described in a broader context of the relation between solutions of the boundary value problem

and that of

$$
\left(p_{0}(\xi) v^{\prime}\right)^{\prime}+\left(p_{1}(\xi) v\right)^{\prime}+p_{2}(\xi) v=0
$$

$$
\left(p_{0}(\xi) w^{\prime}\right)^{\prime}-p_{1}(\xi) w^{\prime}+p_{2}(\xi) w=0
$$

We see that the equation (4.17) and (4.18), (4.24) and derivative of (4.22) are special cases of (6.2) and (6.1).

Suppose we already know a special solution of $(6.1)$, denoted as $v_{0}(\xi)$. Assume this solution satisfies

$$
v_{0}(\xi) \neq 0
$$

We let

$$
w(\xi)=v_{0}(\xi) W(\xi)
$$

This way, we can eliminate $p_{2}$ in (6.2):

$$
\begin{aligned}
0 & =\left(p_{0}(\xi) w^{\prime}\right)^{\prime}-p_{1}(\xi) w^{\prime}+p_{2}(\xi) w \\
& =\left[\left(p_{0} v_{0}^{\prime}\right)^{\prime}+p_{2} v_{0}-p_{1} v_{0}^{\prime}\right] W+\left[2 p_{0} v_{0}^{\prime}+p_{0}^{\prime} v_{0}-p_{1} v_{0}\right] W^{\prime}+p_{0} v_{0} W^{\prime \prime} \\
& =\left[-\left(p_{1} v_{0}\right)^{\prime}-p_{1} v_{0}^{\prime}\right] W+\left[2 p_{0} v_{0}^{\prime}+p_{0}^{\prime} v_{0}-p_{1} v_{0}\right] W^{\prime}+p_{0} v_{0} W^{\prime \prime} \\
& =v_{0}\left(p_{0} W^{\prime}-p_{1} W\right)^{\prime}+2 v_{0}^{\prime}\left(p_{0} W^{\prime}-p_{1} W\right)
\end{aligned}
$$

or simply

$$
v_{0}\left(p_{0} W^{\prime}-p_{1} W\right)^{\prime}+2 v_{0}^{\prime}\left(p_{0} W^{\prime}-p_{1} W\right)=0 .
$$

We integrate Eq. (6.6) once to get

$$
p_{0} W^{\prime}-p_{1} W=C_{1} v_{0}^{-2}
$$

and hence

$$
W(\xi)=C_{2} \exp \left(\int_{0}^{\xi} \frac{p_{1}}{p_{0}} d \eta\right)+C_{1} \int_{0}^{\xi} p_{0}^{-1}(\eta) v_{0}^{-2}(\eta) \exp \left(\int_{\eta}^{\xi} \frac{p_{1}}{p_{0}} d \zeta\right) .
$$

We summarize the above result in the following theorem:

Theorem 6.1. Assume that (6.1) has a solution $v_{0}$ satisfying $v_{0}^{\prime} \neq 0$. Then the general solution of $(6.2)$ is

$$
w(\xi)=v_{0} W(\xi)
$$

where $W(\xi)$ is given by $(6.7)$.

Proof. We already see that the function given by (6.7) are solutions of (6.2) for any constants $C_{1}$ and $C_{2}$. It remains to prove that any solution of $(6.2)$ can be expressed by (6.7) with suitable constants $C_{1}$ and $C_{2}$. Since solutions of (6.2) are uniquely determined by initial data $w(0)$ and $w^{\prime}(0)$, it suffices to prove that for any given initial data $w(0)$ and $w^{\prime}(0)$, there are constants $C_{1}$ and $C_{2}$ such that the $w(\xi)$ given by $(6.8)$ satisfies the initial data. It is clear from (6.7) and (6.8) that $w(0)=v_{0}(0) C_{2}$ or $C_{2}=w(0) / v_{0}(0)$. Similarly, we see that $C_{1}$ can also be determined by $w(0)$ and $w^{\prime}(0)$. This completes the proof. 


\section{REFERENCES}

[AC] S. M. Allen and J. M. Cahn, A macroscopic theory for antiphase boundary motion and its applications to antiphase domain coarsening, Acta Metal. 27, 1085-1095 (1979)

[BES] G. Barles, L. C. Evans, and P. E. Souganidis, Wavefront propagation for reaction-diffusion systems of PDE, Duke Math. J. 61, no. 3, 835-858 (1990)

[BK] L. Bronsard and R. V. Kohn, On the slowness of phase boundary motion in one dimension, Comm. Pure Appl. Math. 43, 983-997 (1990)

[Br] K. A. Brakke, The motion of a surface by its mean curvature, Mathematical Notes, vol. 20, Princeton University Press, Princeton, N.J., 1978

[Ca] G. Caginalp, An analysis of a phase field model of a free boundary, Arch. Rational Mech. Anal. 92, no. 3, 205-245 (1986)

[Ch] X.-Y. Chen, Dynamics of interfaces in reaction diffusion systems, Hiroshima Math. J. 21, 47-83 (1991)

[D] E. De Giorgi, New conjectures on flow by mean curvature, Nonlinear variational problems and partial differential equations (Isola d'Elba, 1990), 120-128, Pitman Res. Notes Math. Ser., vol. 320, Longman Sci. Tech., Harlow, 1995

[E] L. C. Evans, Regularity for fully nonlinear elliptic equations and motion by mean curvature, Viscosity solutions and applications (Montecantini Terme, 1995), 98-133, Lecture Notes in Math., vol. 1660, Springer, Berlin, 1997

[ESS] L. C. Evans, H. M. Soner, and P. E. Souganidis, Phase transitions and generalized motion by mean curvature, Comm. Pure Appl. Math. 45, no. 9, 1097-1123 (1992)

[FH1] H. Fan and J. K. Hale, Large-time behavior in inhomogeneous conservation laws, Arch. Rational Mech. Anal. 125, 201-216 (1993)

[FH2] H. Fan and J. K. Hale, Attractors in inhomogeneous conservation laws and parabolic regularizations, Trans. Amer. Math. Soc. 347, 1239-1254 (1995)

[FHs] P. C. Fife and L. Hsiao, The generation and propagation of internal layers, Nonlinear Anal. 12, no. 1, 19-41 (1988)

[Fife] P. C. Fife, Dynamics of internal layers and diffusive interfaces, CBMS-NSF Regional Conference Series in Applied Mathematics, No. 53, SIAM, Philadelphia (1988)

[FJT] H. Fan, S. Jin, and Z.-H. Teng, Zero reaction limit for hyperbolic conservation laws with source terms, J. Diff. Eqs. 168, 270-294 (2000)

[FJ] H. Fan and S. Jin, Wave patterns and slow motions in inviscid and viscous hyperbolic equations with stiff reaction terms, preprint (2000)

[Har] J. Härterich, Heteroclinic orbits between rotating waves in hyperbolic balance laws, Proc. Roy. Soc. Edinburgh Sect. A 129, no. 3, 519-538 (1999)

[II] T. Ilmanen, Convergence of the Allen-Cahn equation to Brakke's motion by mean curvature, $\mathrm{J}$. Differential Geom. 38, no. 2, 417-461 (1993)

[Lyb] A. N. Lyberopoulos, A Poincarè-Bendixson theorem for scalar conservation laws, Proc. Roy Soc. Edinburgh, 124A, 589-607 (1994)

[Mas] C. Mascia, Traveling wave solutions for a balance law, Proc. Roy. Soc. Edinburgh 127A, 567-593 (1997)

[RSK] J. Rubinstein, P. Sternberg, and J. Keller, Fast reaction, slow diffusion, and curve shortening, SIAM J. Appl. Math. 49, 116-133 (1989)

[Sin2] C. Sinestrari, Asymptotic profile of solutions of conservation laws with source, Differential Integral Equations 9, 499-525 (1996)

[Son] H. M. Soner, Ginzburg-Landau equation and motion by mean curvature. II. Development of the initial interface, J. Geom. Anal. 7, no. 3, 477-491 (1997)

[Sou] P. E. Souganidis, Front propagation: theory and applications, Viscosity solutions and applications (Montecatini Terme, 1995), 186-242, Lecture Notes in Math., vol. 1660, Springer, Berlin, 1997 